\title{
Case report on the use of a functional electrical orthosis in rehabilitation of upper limb function in a chronic stroke patient
}

\author{
Catalin Moghioroiu ${ }^{1,2}$
}

E-mail address: catalinmoghi@yahoo.com

p: $101-111$

\author{
${ }^{1}$ University of Medicine and Pharmacy „Carol Davila” Bucharest / \\ ${ }^{2}$ National Clinical Center for Children Neuro-Psycho-Motor Rehabilitation „Dr. Nicolae Robanescu”
}

\begin{abstract}
Introduction. The increasing incidence of strokes and their occurrence in younger active people require the development of solutions that allow participation, despite the debilitating deficit that is not always solved by rehabilitation. The present report shows such a potential solution.

Objective. In this presentation we will show the effects of using a functional electric orthosis, the high number of repetitions and daily electrostimulation in a young stroke patient with motor deficit in the upper limb, the difficulties encountered in attempting to use orthosis, the results and the course of its recovery over the years.

Materials and Methods. The present report shows the evolution of a 31-year-old female patient with hemiplegia, resulting from a hemorrhagic stroke, from the moment of surgery to the moment of purchasing a functional electrical orthosis and a few months later, highlighting a 3-week period when the training method focused on performing a large number of repetitions of a single exercise helped by the orthosis - 3 weekly physical therapy sessions, with a duration of one hour and 15 minutes, plus 2 electrostimulation sessions lasting 20 minutes each and 100 elbow extension, daily, 6 times a week. The patient was evaluated and filmed at the beginning and end of the 3 week period. The patient's consent was obtained for the use of the data and images presented.

Results. Invalidating motor deficiency and problems specific to the use of upper limb functional electrostimulation in patients with stroke sequelae (flexion synergy, exaggeration of reflex response, wrist position during stimulation, etc.) made it impossible to use orthosis in functional activities within ADL although it allowed the achievement of a single task. Evaluation on the FuglMayer assessment does not show any quantifiable progress, although it is possible to have slightly improved the control of the shoulder and elbow and increased the speed of task execution.

Conclusions. The use of functional orthoses of this type may be useful in patients who still have a significant functional rest in the shoulder, elbow and hand, and where the orthosis can produce an effective grasp. However for some patients, perhaps those who would have been desirable to benefit most from this treatment, the benefit of using this orthosis is minimal.
\end{abstract}

Key words: stroke, upper limb rehabilitation, functional electrostimulation, participation

\section{Introduction}

In general, the possibilities of regaining upper limb (UL) functionality in patients with severe paresis who survive stroke are quite low. Nakayama et al (1994) (1) showing that of the 636 patients included in the study, 214 patients had severe MS paresis at admission ("the arm did not rise against gravity and the fingertips could not reach the palm"), from which only 115 were discharged alive, 99 patients (mean age 78 years) dying during hospitalization. At 64 (56\%) from 115 "UL remain definitively unusable despite intensive and long-term rehabilitation", and only 17 out of 115 had a complete recovery. There was no significant difference regarding sex, age, type of stroke (ischemic or hemorrhagic), time between onset of stroke and hospitalization or duration of hospitalization among patients who recover. Moreover, "the improvement of the UL function observed in 25 of these patients was possible only by compensating with unaffected $U L "$ " they are younger, having a less severe stroke, smaller, subcortically located and a lower impairment of superior cortical functions. The authors point out that it is to be expected that "rehabilitation according to the Bobath technique (which focuses in particular on rehabilitating the affected side) must be expected to have little functional effect on the affected UL in at least half of the stroke patients with severe arm paresis" and, for this reason it would be preferable that learning compensatory techniques be a priority in rehabilitation planning, the more so as "many patients who will experience partial motor recovery will never use the affected UL"

Considering the above, as well as the increasing incidence of strokes and their occurrence in younger, active people, it is necessary to develop performant solutions that will allow participation in activities of daily living (ADL) also for the patients with an invalidating motor deficit for which classic rehabilitation has a reduced functional effect and 
which, for this reason and beyond, will never use the affected UL.

One of the promising solutions, older as a principle, is functional electrical stimulation, basically facilitating muscle contraction by means of electrical impulses in a functional gesture such as walking or grasping. Over the last $10-15$ years, this principle has been integrated into portable devices (functional electric orthosis) that can be purchased by patients and used at home during day-to-day activities.

\section{Electrostimulation}

It is a therapy commonly used in the rehabilitation of UL in patients with stroke hemiplegia, having a relative contraindication in drug controlled epilepsy. Electrostimulation can "reduce disability by improving recovery of volitional movement (therapeutic effect) or by assisting and replacing lost volitional movement (neuroprosthetic effect)" (2). In order to produce effective muscle contractions, peripheral motor neurons need to be intact, which is why it is usually applicable to patients with central motor neuron lesions such as stroke (2) The electrical impulses, most commonly applied by means of surface electrodes attached to the patient's skin, are characterized by frequency, duration and amplitude (intensity). Although the primary purpose of electrostimulation is to train, in the hope that the improvement of functionality will be maintained also when the system is not used, however „for patients who are in the chronic phase of stroke and in whom motor relearning strategies have been exhausted, NMES (neuromuscular electrical stimulation) may be used as a neuroprosthesis. The primary intent of a neuroprosthesis is to enable patients to execute functional tasks with the affected upper limb or walk while using the device as part of routine daily living". (2)

\section{Objective}

In this presentation we will show the effects of using a functional electric orthosis, a large number of repetitions of a single exercise, and daily electrostimulation in a young patient with hemorrhagic stroke and disabling motor of the left UL (no extension movements and insignificant finger flexing), the difficulties encountered in attempting to use orthosis, the results obtained and the course of its rehabilitation over the years.

\section{Materials and Methods}

Case history

The presentation of the following data and images is made by courtesy of the patient who has given her consent to this.

According to the medical letter, the patient aged 31 years suffers a break of an aneurysm of the right communicating posterior artery for which she is transferred to the neurosurgery department with a GCS of 13 points. The cerebral CT shows subarachnoid hemorrhage and ANGIO-CT posterior communicating artery aneurysm in the C7, subarachnoid hemorrhage in the right Sylvian valley, hematic accumulation in the posterior horns of the lateral ventricle, the ventricular system on the median line. The patient is operated in November 2009 (3 days after the stroke) and the surgical cure of the aneurysm is performed. After surgery, develop a left hemiparesis (another stroke?). Post -surgery the patient progression is slow favorable with decompensation at approximately 3 weeks after. CTSCAN indicates the development of an internal hydrocephalus for which reason it is decided to install a ventricular-peritoneal drainage shunt. The patient evolves favorably, receives an indication of mobilization and rehabilitation and is discharged with left hemiparesis diagnosis

In January 2010 begin the rehabilitation program and in about 3 weeks it is able to walk with a four-foot cane. During January 2010 - May 2011 the patient is hospitalized for 3 week periods in several clinics in the country and abroad for rehabilitation (physiotherapy, hydrotherapy, massage) or outpatient in state or private clinics.

At the end of May 2011, the patient is requesting home-based rehabilitation, with the goal of the therapeutic plan to regain motor independence - in terms of walking and left-hand use. At the evaluation, after a year and a half after stroke and about as many months of rehabilitation, it has a 23-point on FuglMeyer (FM) assessment (of 66 possible) with shoulder flexion up to 90 degrees, pronation and supination of the forearm, flexion and the extension of the wrist impossible, but has few degrees of finger flexion. Effort capacity testing (30 squats in 45 seconds) shows a Ruffier index of 6.4. Assessing independence in transfers and walking with the Motor Assessment Scale (MAS) -the first 5 items, shows a score of 28 out of 30 . 
After 4 months of rehabilitation with 3 weekly sessions of classic physical therapy (passive, selfpassive and active, analytic and global mobilizations, postures, slow stretches for spasticity reduction, control and coordination exercises, sensory stimulation, various walking patterns, easy cardio workout etc.) with a duration of 60 minutes each (50 sessions in total) the score on the Fugl-Meyer assessment increases by 3 points from 23 to 26 (improves the shoulder flexion, elbow extension and flexion of the fingers - making possible distal grasp); from supine with the arm $90^{\circ}$ flexed and the elbow extended is possible, with facilitation, the extension of the fingers; the Ruffier index drops to 3.3 indicating a slight increase in effort capacity and slightly increases the walking speed (it can take 4 steps back and forth in 25 seconds vs. 33 at initial evaluation).

In this period, she purchases a functional -drop foot electrostimulation device that he carries while walking outside the house and an electrostimulation device for the UL muscles and starts on her own an intensive stimulation program daily, for the shoulder and for extensors of the wrist and fingers (20 minutes for shoulder and 20 minutes for forearm) in addition to physical therapy sessions.

In the following month, after the electrostimulation program and 23 physical therapy sessions, the FM score remains 26 , but as a result of electrostimulation sessions, the tone of flexion muscles of the wrist and fingers increases slightly. The use of electrostimulation device to facilitate the dorsal flexion of the foot during swing phase improves walking: decreases the excessive compensatory hip flexion, allows a better load on the support foot, balance the steps length and increase walking speed. Very important for increasing independence and participation, given the aspect that the patient is using a walking crutch that keeps busy the healthy hand.

In 2012, continues the home-based rehabilitation program -physical therapy (an average of 2 sessions per week, 92 sessions in total), electrostimulation and massage. The electrostimulation program for the hand extension muscle is reduced, modified and integrated into physical therapy session as follows: 3 short sessions -about 15-20 contractions each (wrist and finger extensions) separated by 5-10 minute periods of light exercises. In order to obtain a contraction as efficiently as possible, in relaxing conditions, the patient is placed in supine with the arm abducted and sustained at 90-degree and forearm pronated. This way of working no longer affects the muscular tone. With the desire to find solutions to help her become more independent she find out at the end of April (2013) about a neuro-electric orthosis that can close and open her hand with electric impulses and which, in theory, will able her to grasp and to move various objects. In order to find out whether or not she can use the orthosis, it is necessary to be evaluated by a specialist to adapt it and show her how it works.

She is asked for a short history of her stroke and rehabilitation program and is asked if he has other medical problems and especially: epilepsy, implanted electronic medical devices (such as pacemaker, etc.), heart problems, surgery at the forearm level and if she is pregnant (which would be an absolute contraindication).

The patient has not made any significant progress over the latest year, despite working constantly, suffers from epilepsy, but with proper treatment is very well-controlled so there has been no seizure in the last year, he has no electronic medical device implanted or surgery at the forearm level has no heart problems and is not pregnant. All that she can do with the UL is to move it from shoulder and elbow, but shoulder flexion at 90 degrees with the elbow extended is impossible, spasticity at the hand flexing muscles can be rated as 1 on Ashworth scale she can make a very low flexion at the thumb and index level, does not have any active extensions even on a single finger, cannot open her hand and cannot use it.

Although, based on what is known today about stroke recovery (1), from experience and in the given situation (reduced proximal control and extreme peripheral deficits), the possibility that the patient can use her hand in the future without the help of the orthosis is almost null, and although it cannot be guaranteed or predicted whether and to what extent there will be an improvement, both the physical therapist and the patient are interested in orthosis not only to be another device of electrostimulation to activate the muscles and to decreases edema, but in especially in the hand become functional again, even with the orthosis on it, meaning it can grab and move objects, take a glass of water, open the door, get dressed (put socks on, close and unclose a button or a zip, tie the laces) etc.

To find out whether the orthosis can help the patient to perform all these things, somewhat unrealistic given the reduced shoulder and elbow control, a test 
is needed to examine the movements that are possible with its help. Even if the muscles are atrophied due to non-use, the orthosis should produce at least wrist and fingers flexion and extension and to some extent allow grasping and moving different objects. Otherwise, just for another electrostimulation device, the costs would not be justified.

In the next month, the patient goes to test the orthosis and purchases it from his own funds.

\section{The orthosis}

AT the present time are available few neuroprostheses with transcutaneous electrodes used to restore functional grasp. Of these, the most marketed; the most present in clinical trials and probably the most known is $\mathrm{H} 200$

H 200 is a 300 gram weight microprocessor based device that provides electrical impulses to stimulate muscle contractions and a rigid plastic orthosis mounted on the forearm. The orthosis has 5 electrodes of different sizes, with textiles surface, the position of which can be adjusted for each individual patient. The electrodes are placed on the surface of the skin over the following muscle: extensor digitorum communis, extensor pollicis brevis, flexor digitorum superficialis, flexor pollicis longus and the thenar muscles (abductor pollicis brevis, flexor pollicis brevis and opponens pollicis) to facilitate as far as possible, closing and opening the patient's hand and basic grasp, including thumb use. The closing and opening command of the hand, to perform functional gestures, is done with a wireless remote.

The orthosis has, according to the manual, 3 programs: functional training, neuromodulation motor and neuroprosthesis. The first program consists of repeated opening and closing of the hand with breaks between movements and is used to train the muscles that act on the wrist and fingers, while the last program has three variants (open hand, grasp and release and key grip) and allows the use of orthosis in functional tasks (such as grabbing and moving different objects), facilitating grasping (by opening and closing the hand) or the lateral grasp of the thumb with the help of a button.

The programs and working time are settled by a trained therapist in accordance with the possibilities and needs of the patient. The patient can choose the working program from those settled and eventually can increase or decrease application intensity.

\section{Intervention}

Taking into account the condition of the patient, the company representative set the orthosis for the following 2 programs:

1. A basic training program (repeated flexions and extensions) lasting 30 minutes.

2. A functional program of this type: open catch and then open - releases which theoretically could be used for a lot of exercises, but in reality it is limited by the actual possibilities of the patient. Excluding the lateral grasp (between thumb and lateral side of the index), probably due to the impossibility of doing it in the present case.

The intensity of the electrical impulse was set 5 (on a scale from 0 to 9 ), approximately $45 \mathrm{~mA}$, according to the technical data sheet of the device, quite a lot but the following aspects must be taken into consideration: the resistivity of the skin and the individual perception differs from one person to another and depending on subcutaneous adipose tissue; position pressure and humidity of the electrodes (to generate conductivity) are also important. Anyway this current intensity was not painful in our case. Due to the orthosis construction, the position and the pressure of the electrodes do not vary much and they must be watered before each use. However, the recommendation is that the intensity be set to the minimum necessary to obtain a proper motor response of the hand with a good fingers extension.

Unfortunately, despite the expectations, the start is modest, as the orthosis fails to produce functional movements during therapy because the abductionadduction of the thumb (an indispensable aspect of effective grasping) is not set due to the impossibility of accomplishment and the incomplete extension of fingers 2-5 (the extension of the metacarpophalangeal joints accompanied by the flexion of proximal and distal interphalangeal joints) makes it impossible to grasp the objects (Fig.2). To exclude that this is due to too much impulse power the application intensity is decreased to 4 . The decreased in intensity slightly improved the extension of the fingers, but functional grasp was still not possible. The further decrease in intensity of value 3 has not brought any benefit. 
In this situation, in order to allow full extension of the fingers, to facilitate the grasp of an object and to generate some functionality the physiotherapist put two simple orthotics on fingers 2 and 3 (Fig. 3). The orthotics were so fixed that, if the force generated by the neuro prosthesis is too large, to be able to detach. With the additional help of this orthotics, the patient manages to grasp and move a small tube of medication, with a diameter about $4 \mathrm{~cm}$. So the lateral grasp is not set, the spherical grasp, the pulp grasp, the tripod grasp and the extension grasp are impossible and the volar grasp is the only one achievable and this with the help of these orthoses. The physiotherapy program continues as before, 2 times a week, including this time and aspects of functional training - grasping and moving the medication tube (2-3 series of 10-15 repetitions) using electric orthoses, but assisted by the physical therapist which triggers the opening and closing command. In addition, the patient uses the orthosis twice a day for 20 minutes for electrostimulation on the flexor and extensor muscles of the hand (40 minutes daily).

After one month, during which both the patient and the physical therapist get used to the orthosis, in the hope of significant progress and to highlight the effects of this work modalities, a new Fugl -Mayer assessment is performed, the patient is filmed again, and it's starts $a$ 3-week training period with a greater number of repetitions: 3 physical therapy sessions weekly, approximately one hour and 15 minutes each, in which one exercise will be perform, grasping and moving an small object (medication tube), with the affected hand and with the help of the orthosis, 160 times per session. The session is preceded by the execution of several active mobilizations of the trunk and passive mobilizations of the affected UL performed by the patient before the physical therapist arrives. Exercises are split into 4 series of 40 repetitions, separated by 2 minute breaks. At 10 repetitions in each series is used the healthy hand, which brings to the middle line of the body a container (a cup) in which the plastic tube is placed on each repeat, hoping for further progress due to the bilateral training. To these exercises is added 2 daily electrostimulation sessions with the orthosis, 20 minutes each , separately done by the patient (about 400 flexions and extensions daily, lasting 6 seconds each) and 100 elbow extensions from side lying, maintaining, as much as possible the 90 -degree arm abduction, separated by breaks at choice, daily, 6 times a week. At the end of the 3 weeks period, we re-evaluated and filmed the patient.

The reason for choosing this treatment modalities was, on one hand, classical physical therapy did not bring significant progress, on the other hand are enough studies $(2,4,5)$, arguments $(2,5)$ and recommendations $(2,6)$ in the literature that the rehabilitation should be oriented on the repetition, in large numbers of functional tasks $(4,6)$.

Assessments used

To assess the control and coordination at UL level, in order to notice the progress achieved after the intervention, we used the Fugl-Meyer Assessment (7), a 22-item scale, noted from 0 , cannot perform to $2=$ can perform fully, having a maximum score of 66 points. The assessment has the advantage of evaluating control and coordination at the shoulder, elbow, wrist and hand in both inside and outside flexion synergies.

For spasticity evaluation we used the Modified Aschworth Scale (MAS), which is perhaps the most known and the most used scale for assessing spasticity, although both the way of assessment and the relationship between spasticity and disability after stroke are being questioned in recent years $(8,9,10$, 11)

\section{RESULTS}

The patient performed all scheduled sessions and exercises. Therapy has not caused discomfort, fatigue, or pain, except for a single situation where the intensity of the stimulus has generated pain, the slight change in the orthosis position and the reduction in intensity have removed this problem which did not appear later not even at that intensity.

The evaluation on the FM scale does not show any progress, although it is possible to slightly improved the shoulder and elbow control, unquantifiable with this scale

The distance between the tip of the thumb and the index, with the relaxed hand at rest, increased by several mm (Fig. 5) possible due to the elongation of the soft tissue due to the stretching performed following electrostimulation with the orthoses on the fingers or possibly due to the decrease of the muscular tone at rest. Lengthening flexor muscles or possibly decreasing the tendon reflex responsiveness makes the extension produced by orthotics at the end of the 
3 weeks of therapy no longer producing such a strong flexion of proximal and distal interphalangeal joints of fingers 2-5 together with the extension of the metacarpophalangeal joints, even with the intensity set to 5 .

The evaluation of spasticity using the MAS scale does not show any quantifiable changes

The muscular force may have increased slightly, but in the absence of adequate equipment, this has not been measured.

The speed of execution of the tasks may have increased also, but this has not been evaluated as well. To exemplify the results, we will show below some images. All pictures are taken at the maximum opening amplitude of the hand (frame stop on film)

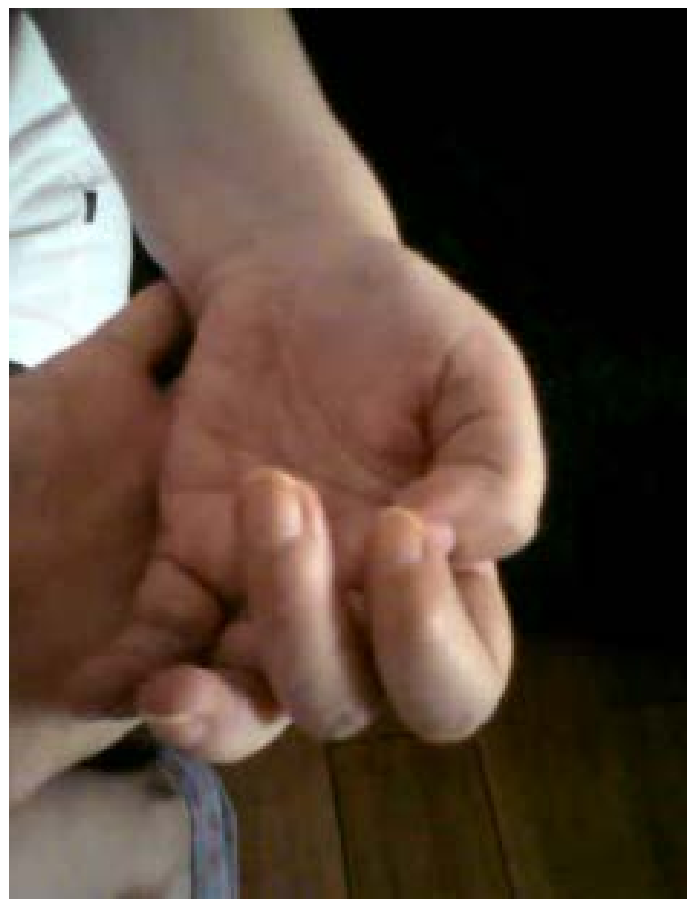

Fig. 1

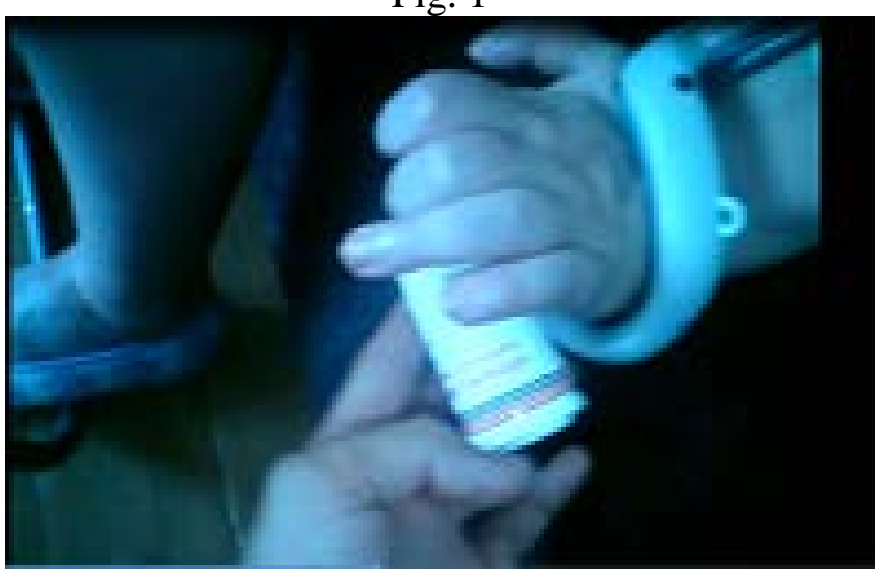

Fig.2
Fig. 1 Hand of the patient at the time of the orthosis acquisition (one month before intense working program). The patient was asked to keep her hand relaxed -it may be seen the slight increase in tone of the following muscle: flexor digitorum superficialis, flexor pollicis longus, flexor pollicis brevis, flexor carpi ulnaris, flexor carpi radialis

Fig.2 The patient's attempt to catch the plastic tube with the hand open by means of the electric impulse (wrist and finger extension command) is without result, as fingers 2 and 3 do not extend to grasp the object.

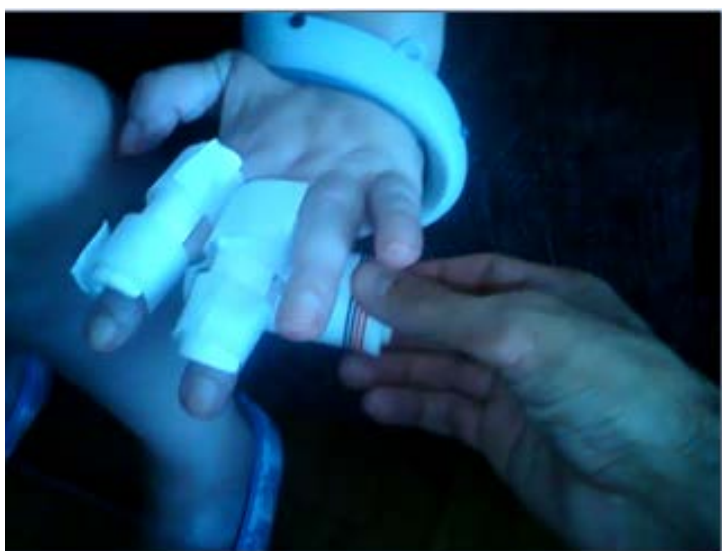

Fig.3

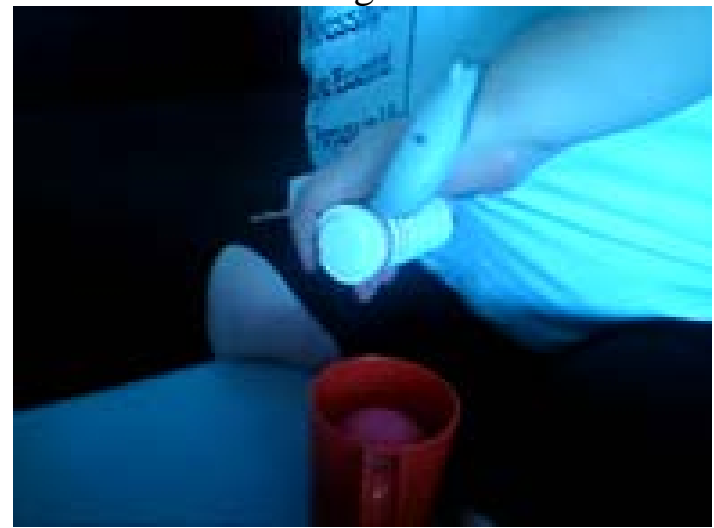

Fig.4

Fig.3 The hand of the patient opened with the orthosis one week after its purchase. It can be seen the extra help offered by the orthoses of the fingers both in the opening of the fingers (almost complete extension) (Fig.3) and in the grasping of the object (Fig.4). Also, probably due to the anatomy of the hand (12), the fitting of the orthoses facilitates the extra extension of the fingers 4-5.

Fig.4. Functional exercise which was the basis of the therapy during the 3 weeks of intense work program. Grasp of the plastic tube facilitated by fingers orthoses. 


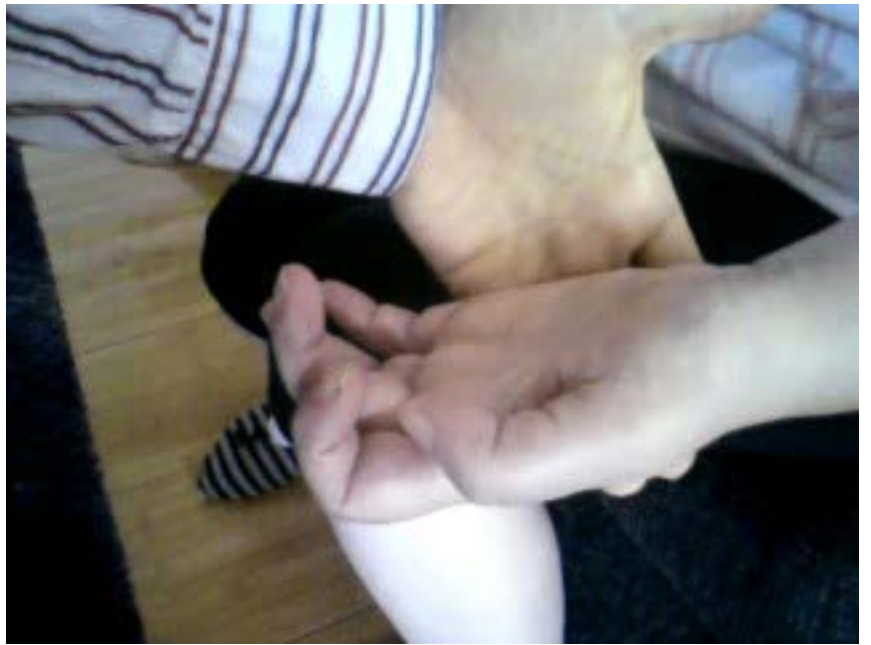

Fig 5

Fig. 5 The hand of the patient at the end of the 3 weeks of intense work program. The patient was asked to hold her hand relaxed - it may observe the slight elongation / decrease of the tone of the following muscle: flexor digitorum superficialis, flexor pollicis brevis, flexor carpi ulnaris and flexor carpi radialis, the muscle that have benefited from stretching during electrostimulation with orthosis on the fingers and not the flexor pollicis longus that was not stretched during exercises.

After this period, the patient continues with the 40minute electrostimulation program daily and 1-2 weekly physical therapy sessions, interleaved by treatment periods (including physical therapy) in different clinics. The physical therapy program is about the same as before this period (including only 2-3 sets of 10-15 repetitions of the functional exercise with the affected hand).

At six months after the 3 weeks of intense work program the results of physical therapy and electrostimulation were the same.

\section{Discussions}

This presentation highlights the effect of intense electrostimulation and especially functional electrostimulation with a high number of repetitions in the case of a patient with a chronic stroke and a major deficit to the left upper limb.

The treatment methodology used in this case aimed to put into practice the world wide proven efficiency methods used in the rehabilitation of upper limb in stroke patients, in the hope that progress will be made, especially as classical physical therapy, in this case, has obtained modest results.
To participate in everyday activities, efficient grasp remains an indispensable motor function. In practice, it has been demonstrated (12) that it is possible to perform the basic functional grasps with the help of electrostimulation by accurately placing several pairs of electrodes, but this goal is difficult to achieve within a neuroprosthesis. In addition, electrical stimulation for restoration of grasping is limited by undesirable movements and discomfort (12) „Ideally ES (electrical stimulation) systems and their associated electrodes should... be able to selectively activate the target motor neurons, whilst remaining simple to configure and comfortable to use" but just „the inability to selectively activate target muscles (resulting in unwanted movements) and the need to configure and accurately place multiple sets of electrodes" are their main limitations (12)

Although functional grasps are easy to perform volitionally, „functional restoration using transcutaneous electrical stimulation is complicated by the lack of selective muscle activation..., deep motor neurons (and hence muscles) cannot be activated without first co-activating any superficial nerves (including nociceptors) or motor neurons producing unwanted movements and increasing discomfort” (12). For example „unwanted wrist flexion can be caused by simultaneous co-activation of superficial FCR (Flexor carpi radialis) and FCU (Flexor carpi ulnaris) muscles" (12). In addition, forearm rotation and excessive muscle contractions can cause the underlying muscles to move relative to the surface of the skin, thereby affecting selective muscle activation and requiring changing the position of the transcutaneous electrodes according to the orientation of the arm (12). It is necessary, therefore, to take into account that the exact position and orientation of the sets of electrodes depend on the individual requirements of the subject (12). „Integrating multiple electrodes and connecting wires into a garment or orthosis (e.g. H200) can help simplify the application of the neuroprosthesis, but does not improve selective activation of functional grasps (12)"

Thus, although the H200 is the most marketed, most present in clinical trials, and probably the most known functional electric orthosis currently used to restore functional grasp, although it has the advantage of maintaining constantly the position of the electrodes after initial determination, a time consuming and error prone aspect to other electrostimulation 
modalities (12),thus facilitating its use by the patient without the therapist's help, although it allows achieving a large number of repetitions of taskoriented activities, an indispensable aspect to stimulating cortical changes that lead to functional gains in order to progress and maintain it after the therapies are completed (3) although it has proved its efficiency in a series of high quality clinical trials (Page et al., Santos et al., etc.) it still has a number of limitations and disadvantages, aspects also observed in our situation:

\section{Limitations and disadvantages related to the construction of the orthosis}

Although "for a patient with no wrist or finger AROM (Active Range of Motion), NMES (neuromuscular electrical stimulation) does not provide support of the wrist into extension to facilitate the optimum reach, grasp, and release movement necessary to perform a number of task-specific activities" (13), the blocking of the wrist movements is far from desirable (12), especially because of the importance this movement has for grasping and using objects (14). In this regard, Cameron et al (1999) demonstrated that, although applying an electrode over the common extensor muscle of the fingers is sufficient to produce a complete opening of the hand to the hemiplegic patient with the aid of functional electrical stimulation; this opening "strongly depends on wrist posture". Thus "maximal hand opening occurred when the patients were fully relaxed with wrists in the fully flexed position.... as the wrist was extended, hand aperture achieved by electrical stimulation progressively declined, reaching zero at 40 degrees of wrist extension" (15).

In addition, the electrode support for the thenar muscle group prevents effective grasping (14), exactly the aspect for which this neuroprosthesis is intended, considering the fact that "numerous objects manipulated during daily life (e.g., coffee cups, bottles, spoons, or pencils), require successful movement of the thumb to form a functional grip....the more so as ...some patients suffer from involuntarily enlarged flexor activity, which hampers extension of individual fingers and therefore the release of objects"That is why ,selective finger extension (to counteract enlarged flexor activity) and thumb opposition are the focus when developing effective tools for relearning grasp and release functions"
(16). Then the fact that "the electrodes do not allow selective muscle activation, resulting in unwanted wrist flexion which is physically restrained by the rigid orthosis" is another problem of $\mathrm{H} 200$ - but ,the use of a rigid orthosis to restrain unwanted movements is far from desirable (12)"

An alternative solution would be to apply a multi-pad / multi-channel stimulation system that would make it easier to identify the appropriate position of the electrodes and provide the possibility of selective stimulation of different muscle groups $(12,14)$. However, as the creator of the technology mentioned, „the size and complexity of the control systems are unsuitable (yet) for producing a portable neuroprostheses for use in the home" and further requires a full functional assessment for use in $A D L$ " (12)

A phenomenon that occurs in the hemiplegic patient and worsens his condition is the presence of flexion synergy (involuntary co-activation along several joints). For example, "when a stroke patient abducts and/or flexes his or her shoulder (as when reaching), the biceps and forearm flexors may involuntarily activate (flexor synergy) at the same time, preventing lifting the arm and reaching forward simultaneously" (17). In practice, it has been observed that the electric stimulation of the finger extensors can produce the functional opening of the hand when the stroke patient is relaxed but if the patient "exerts the effort to reach or open the hand during stimulation, the hand does not open as much as when the person remains relaxed"the electrical stimulation being defeated by the co-activation / co-contraction of the flexor fingers (17). In addition to co-activation and co-contraction, reflex exaggeration could also limit the opening of the hand, as they are known to increase after a stroke, the stimulation of the extensor digitorum communis can elicit finger flexor stretch reflexes (17)

In addition, for the orthosis to be functional, it is also necessary to have some control at the shoulder and the elbow level (8), at least to reach with the hand above the object, hold the hand in this position to grasp and retract the hand with the object caught.

Another problem that lowers the efficiency of using the H200 is that the closing and opening signal of the hand is given by the wireless remote which means that the healthy hand will not be able to simultaneously participate in the activities that require both hands. In addition, as Hara (2008) 
mentioned, "it is tiresome to manipulate the switch in the open-loop control system with unaffected upper limb during FES interventions” (18)

\section{Limitations observed in clinical trials}

First and perhaps the most important aspect -for its use it requires also a good control of the shoulder and elbow, an aspect seen in very few hemiplegic patients (1), then a low degree of peripheral spasticity, mobility at the wrist and fingers level, and even some peripheral (hand and fingers) control. In this regard, patients who achieved significant results in clinical trials -either were in acute phase of a stroke (2 to 4 weeks after Ring and Rosenthal (2005)) either in post-acute phase (3 to 6 months after stroke Alon et al (2007))

or, if they were in the chronic phase of stroke (over 6 months Page et al (2012)) had to have at least a partial capacity to move outside of synergies at the affected elbow and not to have a higher score (more than 3) on Modified Ashworth Scale at the elbow, wrist and fingers level. More than that, most of the 4.2 points progress on FM (P_.0007) in this study was due to improving the functionality of the shoulder, forearm and wrist, aspects that already had better functionality. The authors noting that up to that time, for this type of patients and for a treatment time of less than 120 minutes, "no other study group exhibited significant FM changes after intervention" and that "given the chronic and moderately impaired nature of (the) sample, some of the FM increases may be attributable to peripheral soft tissue changes rather than (or in addition to) central nervous system changes". In addition, it is worth noting that although the inclusion criterion in this study was the absence of active wrist and fingers extension, neuroprosthesis has managed to generate an efficient grasp for these patients enough to allow them to participate in complex tasks of daily activities as would be to drink from a cup or to hold a key between the index and the thumb (4)

or, in another study (stroke older than 3 years but on average 6.1 years Santos et al (2006) had to have at least $10^{\circ}$ of passive extension of the wrist and at least $90 \%$ of the passive extension of fingers and thumb with the wrist in neutral position, active shoulder flexion of at least $30^{\circ}$, and active elbow extension of $10^{\circ}$ from $90^{\circ}$ of flexion. In this study the taskoriented training method has brought an increase in performance on the Fugl-Meyer scale of 6.2 (p $<0.014)$ versus $5.4(p<0.018)$ in the simple electrostimulation program, the results diminishing 10 days post intervention to 4.0 and respectively 3.6 points, the authors noting that "retaining therapeutic benefit from NMES may indeed be related to severity, the time interval from the initial injury until voluntary muscle activation occurs, or other factors", and that "chronic stroke survivors exhibit 'functional disuse syndrome' which can benefit from 'forced use' which is highlighted by the fact that "despite that individual subjects had relatively low function in the hand subtests of the FM test, they were able to perform fairly well on the JHFT tests at baseline" (JebsenHand Function Test is a test that measure performance speeds for skilled dexterity tasks) and that „moderate functional ability was observed in our group for the tasks that accommodated movements performed within their (patients) available range of motion"Moreover, the fact that for a subject it was not possible to "electrically activate the wrist extensors comfortably in order to overcome the flexor spasticity/tone at the hand" and that this subject „was unable to perform the task of grasping or releasing the ball during the active intervention" shows that there are chronic stroke patients who cannot benefit from this type of intervention (19)

For scientific rigor, however, it is worth mentioning the important difference between the number of patients, the method used and the time allocated to therapy in the two studies as follows: the first study on 32 patients (Page et al 2012) spread over a period of 8 weeks, a total of 40 sessions, 120 minutes each, oriented on functional tasks from ADL and the second study on 8 patients (Santos et al 2006) over a 2-week period, a total of 10 sessions, 30 minutes each, in which the task that patients had to execute it, with the help of the neuroprosthesis consists in grasping and releasing a tennis ball.

However, although unexpectedly, there is no significant difference between the results obtained in the two studies on the FM scale 4.2 versus 4 points (at 10 days post intervention) 


\section{CONCLUSIONS}

Invalidating motor deficiency with significant impairment of control and coordination at the shoulder and elbow level, together with the problems specific to the use of functional electrostimulation in the upper limb in stroke patients (flexion synergy, exaggerating reflex response, wrist position during stimulation etc.) made it impossible to use the neuroprosthesis in functional activities within ADLs although, after attaching two orthotics for fingers, allowed accomplishment of a task.

In the case of our patient, the use of this type of neuroprosthesis did not bring the expected benefit, neither during the treatment session and much less after. The result on the Fugl-Meyer scale remained unchanged after 3 weeks of intense therapy and, from this point of view, at 6 months the results of physical therapy and electrostimulation were the same.

Therefore, although the use of this type of neuroprosthesis presents a number of advantages, the most important one is the fact that facilitate grasping and implicitly participation for a number of patients with an important functional rest at the shoulder, elbow and hand, patients in whom the orthosis succeeds in producing effective grasping, there still are some patients, perhaps those who would have been desirable to benefit the most from this type of treatment, for which the benefit of using this neuroprosthesis is minimal. Considering the fact that, for these patients, neither classical therapy methods have proven their effectiveness, it is necessary to explore more efficient and effective alternative solutions.

\section{Bibliography}

1. Compensation in recovery of upper extremity function after stroke: the Copenhagen Stroke Study Nakayama H, Jorgensen HS, Raaschou HO, Olsen TS (1994). Arch Phys Med Rehabil 75(8): 852-7

http://www.archives-pmr.org/article/00039993(94)90108-2/pdf

2. "Neuromuscular Electrical Stimulation for Motor Restoration in Hemiplegia” Jayme S. Knutson, Michael J. Fu1, Lynne R. Sheffler and
John Chae Phys Med Rehabil Clin N Am. 2015

November ; 26(4):

729-745.

doi:10.1016/j.pmr.2015.06.002.

https://www.ncbi.nlm.nih.gov/pmc/articles/PMC463 0679/

3. Technology for rehabilitating the upper limb after stroke: the hand Jon Graham British Journal of Neuroscience Nursing Volume 10, Issue Sup2 Published Online: May 05, 2014

http://trishakoerkenmeier.musites.org/wpcontent/uploads/2017/04/Technology-forrehabilitating-the-upper-limb-after-stroke.pdf

4. Longer Versus Shorter Daily Durations of Electrical Stimulation During Task-Specific Practice in Moderately Impaired Stroke Stephen J. Page, PhD, MS, S/OT, Linda Levin, PhD, Valerie Hermann, MS, OTR/L, Kari Dunning, PhD, PT, Peter Levine, BA, PTA Arch Phys Med Rehabil 2012;93:200-6.

http://www.archives-pmr.org/article/S00039993(11)00850-1/fulltext

https://era.library.ualberta.ca/files/n870zr301

5. Remodeling of cortical motor representations after stroke: implications for recovery from brain damage RJ Nudo 1997 - nature.com

https://www.nature.com/articles/4000188

6. Principles of Experience-Dependent Neural Plasticity: Implications for Rehabilitation After Brain Damage Jeffrey A. Kleim and Theresa A. JonesJournal of Speech, Language, and Hearing Research, February 2008, Vol. 51, S225-S239. doi:10.1044/1092-4388(2008/018)

https://jslhr.pubs.asha.org/article.aspx?articleid=177 3394

7. https://neurophys.gu.se/digitalAssets/1520/1520 603_fma-ue-protocol-english-updated20150311.pdf

8. Two new dynamic devices / orthoses for training / assisting / rehabilitation of hand functionality in patients with stroke as a result of clinical experience, Catalin Moghioroiu, Balneo Research Journal DOI: http://dx.doi.org/10.12680/balneo.2016.133

Vol.7, No.4, December, 2016 143-150

http://bioclima.ro/Balneo133.pdf

9. Stop using the Ashworth Scale for the assessment of spasticity Judith F M Fleuren, Gerlienke E Voerman, Catelijne V Erren-Wolters, Govert J Snoek, Johan S Rietman, et al... Journal of Neurology, Neurosurgery and Psychiatry, BMJ 
Publishing Group, 2009, 81 (1), pp.46. $<10.1136 /$ jnnp.2009.177071>.<hal-00552783> https://hal.archives-ouvertes.fr/hal-

00552783/document

10. Stop using the Ashworth scale for the assessment of spasticity Sunnerhagen, Katharina S. Journal of Neurology, Neurosurgery and Psychiatry; London Vol. 81, Iss. 1, (Jan 2010): 2.

https://scholar.google.ro/scholar?hl=en\&as_sdt=0\% 2C5\&q=Stop+using+the+Ashworth+scale+for + the+assessment + of + spasticity $+\& b$ tnG $=$

11. Ashworth Scales are unreliable for the assessment of muscle spasticity Noureddin Nakhostin Ansari, PhD PT, Soofia Naghdi , MSc PT, Hoda Moammeri , BSc PT \& Shohreh Jalaie MSc https://doi.org/10.1080/09593980600724188

Pages 119-125 | Accepted 28 Apr 2005, Published online: 10 Jul 2009v Physiotherapy Theory and Practice Volume 22, 2006 - Issue 3 https://scholar.google.ro/scholar?hl=en\&as_sdt $=0 \%$ 2C5\&q=Ashworth+Scales+are+unreliable + for + the+assessment + of + muscle+spasticity\&btnG $=$

12. Transcutaneous electrode technology for neuroprostheses Marc Lawrence Doctoral Thesis 2009

https://doi.org/10.3929/ethz-a-005828341,

13. A four-week, task-specific neuroprosthesis program for a person with no active wrist or finger movement because of chronic stroke $K$, Berberich A, Albers B, et al. Phys Ther. 2008;88:397-405.]

https://academic.oup.com/ptj/article/88/3/397/27424 26

14. Multi-Pad Electrode for Effective Grasping: Design Lana Popović-Maneski, Miloš Kostić, Goran Bijelić, Thierry Keller, Sindi Mitrović, Ljubica Konstantinović, and Dejan B. Popović IEEE TRANSACTIONS ON NEURAL SYSTEMS AND REHABILITATION ENGINEERING, VOL. 21, NO. 4, JULY 2013 https://www.researchgate.net/profile/Dejan_Pop ovic4/publication/236080815_MultiPad_Electro de_for_Effective_Grasping_Design/links/56669 3a908ae15e74634d3bc/Multi-Pad-Electrodefor-Effective-Grasping-Design.pdf

15. The effect of wrist angle on electrically evoked hand opening in patients with spastic hemiplegia T. Cameron ; K. McDonald ; L. Anderson ; A. Prochazka, Published in: IEEE Transactions on
Rehabilitation Engineering ( Volume: 7, Issue: 1, Mar 1999 ) Page(s): 109 - 111 DOI: 10.1109/86.750560

https://scholar.google.ro/scholar?hl=en\&as_sdt= $0 \% 2 \mathrm{C} 5 \& \mathrm{q}=$ The + effect + of + wrist + angle + on + ele ctrically+evoked+hand+opening + in + patients $+w$ ith + spastic + hemiplegia\&btnG $=$

16. Selectivity and Resolution of Surface Electrical Stimulation for Grasp and Release Ard J. Westerveld, Alfred C. Schouten, Peter H. Veltink, Senior Member, IEEE, and Herman van der Kooij IEEE TRANSACTIONS ON NEURAL SYSTEMS AND REHABILITATION ENGINEERING, VOL. 20, NO. 1, JANUARY 2012 https://scholar.google.ro/scholar?hl=en\&as_sdt= 0\%2C5\&q=12.\%09Selectivity+and+Resolution + of + Surface+Electrical+Stimulation+for+Grasp + and + Release $+\& b t n G=$

17. Functional Electrical Stimulation to augment poststroke reach and hand opening in the presence of voluntary effort: a pilot study Nathaniel S. Makowski1,2, Jayme S. Knutson, Ph.D.1,2,3, John Chae, M.D.1,2,3, and Patrick E. Crago, Ph.D.1,2 Neurorehabil Neural Repair. 2014 ; 28(3): 241-249. doi:10.1177/1545968313505913.

https://www.ncbi.nlm.nih.gov/pmc/articles/PMC412 8408/

18. Neurorehabilitation with new functional electrical stimulation for hemiparetic upper extremity in stroke patients Y Hara - Journal of Nippon Medical School, 2008 - jstage.jst.go.jp https://scholar.google.ro/scholar?hl=en\&as_sdt=0\% 2C5\&q=14.\%09Neurorehabilitation+with+new + functional+electrical+stimulation+for+hemipar etic+upper+extremity+in+stroke+patients $+\& b t n$ $\mathrm{G}=$

19. Neuromuscular electrical stimulation improves severe hand dysfunction for individuals with chronic stroke: a pilot stud. Santos M, Zahner LH, McKiernan BJ, et al. J Neurol Phys Ther. 2006;30:175-183.

20. https://journals.lww.com/jnpt/Fulltext/2006/120 00/Neuromuscular_Electrical_Stimulation_Impr oves.2.aspx 